\title{
Prevalência de anemia e determinantes da concentração de hemoglobina em gestantes
}

\section{Prevalence of anemia and determinants of hemoglobin concentration in pregnant women}

\author{
Elma Izze da Silva Magalhães ${ }^{1}$, Daniela Santana Maia ${ }^{2 \dagger}$, Michele Pereira Netto ${ }^{3}$, \\ Joel Alves Lamounier ${ }^{4}$, Daniela da Silva Rocha ${ }^{5}$
}

\begin{abstract}
Resumo
Introdução: A anemia é caracterizada pela reduzida concentração de hemoglobina e, durante a gestação, está associada à maior morbimortalidade fetal e materna. Objetivo: Avaliar a prevalência de anemia e os fatores determinantes da concentração de hemoglobina em gestantes. Método: Estudo transversal com uma amostra de 328 gestantes atendidas nas unidades de saúde urbanas de Vitória da Conquista, na Bahia. Foram realizadas a aplicação de questionário, a avaliação antropométrica e a coleta de sangue por punção capilar para dosagem de hemoglobina em $\beta$-hemoglobinômetro portátil. Foram consideradas anêmicas as gestantes com hemoglobina $<11 \mathrm{~g} / \mathrm{dL}$. Os determinantes da concentração de hemoglobina sérica foram identificados por meio da regressão linear múltipla. Resultados: A prevalência de anemia foi de 18,9\%, e a média de hemoglobina, de 11,9 g/dL (desvio-padrão: 1,2). Foram observadas menores médias de concentração de hemoglobina entre as gestantes que iniciaram o pré-natal no segundo trimestre ( $\beta$ : -0,28; IC95\%: -0,54 a -0,02) e que não usavam suplemento de ferro $(\beta:-0,51 ;$ IC95\%: $-0,79$ a $-0,23)$, enquanto a maior média foi verificada entre as mulheres primigestas ( $\beta: 0,34$; IC95\%: 0,06 a 0,62). Conclusão: $A$ anemia nas gestantes avaliadas é um leve problema de saúde pública, e as concentrações de hemoglobina foram associadas aos fatores obstétricos e à assistência pré-natal.
\end{abstract}

Palavras-chave: anemia; hemoglobina; gestante; assistência pré-natal; sais de ferro.

\begin{abstract}
Background: Anemia is characterized by reduced hemoglobin concentration and, during pregnancy is associated with increased fetal and maternal morbidity and mortality. Objective: To evaluate the prevalence of anemia and the determinants factors of hemoglobin concentration in pregnant women. Methods: Cross-sectional study with a sample of 328 pregnant women patients of the urban Health Units of Vitória da Conquista, Bahia. We applied a questionnaire, anthropometric evaluation, and collected blood by capillary puncture for dosage of haemoglobin using a portable $\beta$-hemoglobinometer. Were considered anemic those pregnant women with hemoglobin $<11 \mathrm{~g} / \mathrm{dL}$. The determinants of serum hemoglobin concentration were identified through multiple linear regression. Results: We observed anemia in $18.9 \%$ of the participants, and the mean hemoglobin concentration was $11.9 \mathrm{~g} / \mathrm{dL}$ (standard deviation: 1.2). We observed lower mean of hemoglobin concentration among pregnant women who started prenatal care in the second trimester ( $\beta$ : $-0.28 ; 95 \% \mathrm{Cl}:-0.54$ to -0.02 ) and who did not used iron supplementation ( $\beta$ : $-0.51,95 \% \mathrm{Cl}:-0.79$ to -0.23 ), while a higher mean was observed among primigavidae women ( $\beta: 0.34,95 \% \mathrm{Cl}: 0.06$ to 0.62$)$. Conclusion: Anemia in the population evaluated is a mild public health problem, and hemoglobin concentrations were associated to obstetric factors and prenatal care. Keywords: anemia; hemoglobin; pregnant women; prenatal care; iron salts.

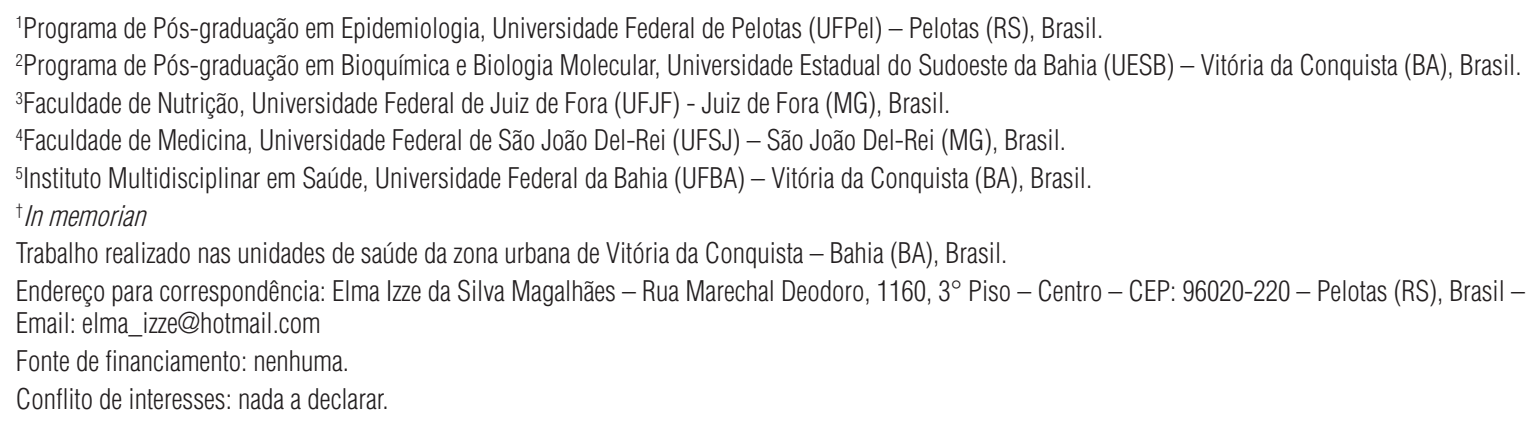




\section{INTRODUÇÃO}

A anemia é caracterizada pela redução na concentração de hemoglobina ${ }^{1,2}$. Para a biossíntese de hemoglobina, as hemácias em desenvolvimento requerem quantidades adequadas de ferro, protoporfirina e globina; dessa forma, as anemias recebem diferentes classificações, dependendo de qual dos compostos está deficiente ${ }^{1}$.

A anemia por deficiência de ferro é a forma mais comum, sendo responsável por aproximadamente $90 \%$ de todos os tipos de anemia no mundo ${ }^{3}$, e ocorre como resultado de diversos fatores, especialmente da ingestão e/ou da absorção deficiente de ferro e do aumento do volume sanguíneo' ${ }^{1}$.

As gestantes têm maior risco de desenvolvimento da anemia devido à elevada necessidade de ferro, determinada pela rápida expansão dos tecidos e da produção de hemácias, difíceis de serem supridas apenas pela dieta ${ }^{4}$. A anemia durante o período gestacional está associada à maior morbimortalidade fetal e materna, bem como ao maior risco de parto prematuro e baixo peso ao nascer, que, por sua vez, associam-se a riscos aumentados de infecções e de mortalidade infantil ${ }^{5,6}$.

Segundo estimativas da Organização Mundial de Saúde $(\mathrm{OMS})^{7}$, a prevalência global de anemia em gestantes entre 1993 e 2005 correspondeu a 41,8\%, sendo classificada como um grave problema de saúde pública. No Brasil, contudo, ainda não há dados consistentes que permitam estimar com precisão a prevalência de anemia na gestação em âmbito nacional ${ }^{8}$. Côrtes et al. ${ }^{9}$, ao avaliarem os estudos sobre a prevalência de anemia ferropriva em gestantes realizados entre 1970 e 2005 no Brasil, retrataram uma escassez de trabalhos com esse grupo populacional, especialmente a partir dos anos 2000, observando-se, em contrapartida, o foco das pesquisas no grupo infantil.

Nesse contexto, o objetivo deste estudo foi avaliar a prevalência de anemia e os determinantes da concentração de hemoglobina em gestantes atendidas nas unidades de saúde da zona urbana do município de Vitória da Conquista, na Bahia.

\section{MÉTODO}

Trata-se de um estudo transversal, realizado em todas as unidades de saúde da zona urbana de Vitória da Conquista, compreendendo 15 unidades de saúde da família, 3 policlínicas de atenção básica e 3 centros de saúde, que oferecem assistência pré-natal às gestantes estudadas.

A cidade de Vitória da Conquista, situada na região sudoeste da Bahia, é o terceiro maior município do Estado, tendo sua economia baseada, principalmente, no setor de serviços ${ }^{10}$. Segundo dados do Instituto Brasileiro de Geografia e Estatística (IBGE), em 2010, o município possuía 306.866 habitantes e um índice de desenvolvimento humano (IDH) de $0,678^{11}$. Em relação ao aspectos epidemiológicos e de saúde do município, no ano de 2009, o percentual de internação relacionado à gravidez e ao puerpério em Vitória da Conquista foi de 72,8 e 48,3\% na população de mulheres na faixa etária de 15 a 19 anos e de 20 a 49 anos, respectivamente. Nesse mesmo ano, o percentual de cobertura de consultas pré-natal foi equivalente a $86,8 \%{ }^{12}$.

A amostra foi calculada com o auxílio do programa Statcalc do software Epi-info 6.04, considerando o número total de gestantes atendidas nas unidades de saúde da zona urbana de Vitória da Conquista entre 2009 e 2010 ( $n=2.316)$, uma prevalência de anemia estimada em $40 \%{ }^{13}$, com precisão de $5 \%$ e intervalo de confiança de 95\%, resultando em um tamanho amostral mínimo de 319 gestantes. Como critério de inclusão, a gestante deveria ter idade igual ou superior a 18 anos e ausência de alterações de saúde que pudessem interferir na concentração de hemoglobina, que não a anemia. Foram excluídas as gestantes com alguma incapacidade cognitiva para responder ao questionário do estudo.

A coleta de dados ocorreu no período de maio de 2010 a junho de 2011, por meio do agendamento prévio nas instituições de saúde nos dias de atendimento pré-natal. As gestantes foram convidadas a participar do estudo de forma aleatória, sendo informado o objetivo da pesquisa e solicitada a assinatura do Termo de Consentimento Livre e Esclarecido, caso concordassem em participar do estudo.

Os dados foram coletados por alunos do curso de Nutrição, previamente treinados para aplicação do questionário e para coleta de sangue por punção digital e das medidas antropométricas.

As informações foram coletadas por meio da aplicação de um questionário, incluindo questões referentes aos dados socioeconômicos e demográficos, às características obstétricas, comportamentais e antropométricas. Além disso, obtiveram-se informações sobre frequência de consumo de alimentos fontes de ferro e suplementação com sais de ferro. $\mathrm{O}$ instrumento foi elaborado pelos pesquisadores e, embora não validado, foi testado em um estudo-piloto realizado antes da coleta de dados.

Para determinação da concentração sérica de hemoglobina, uma amostra de sangue foi coletada em microcuvetas descartáveis por punção capilar, sendo realizada a dosagem de hemoglobina em $\beta$-hemoglobinômetro portátil (Hemocue ${ }^{\circledR}$ ). Para o diagnóstico de anemia, adotou-se como ponto de corte a concentração de hemoglobina inferior a $11,0 \mathrm{~g} / \mathrm{dL}$, de acordo com o preconizado pelo Ministério da Saúde ${ }^{14}$.

Para o cálculo da idade gestacional, foi utilizada a data da última menstruação registrada no cartão da gestante.

Os dados antropométricos de peso e de altura foram coletados segundo as técnicas recomendadas pelo Sistema de Vigilância Alimentar e Nutricional (SISVAN) ${ }^{15}$. Para obtenção do peso corporal, utilizou-se balança digital eletrônica portátil Marte ${ }^{\circledR}$ com capacidade de $200 \mathrm{~kg}$ e sensibilidade de $50 \mathrm{~g}$. A altura foi verificada utilizando-se estadiômetro portátil Alturexata ${ }^{\circledR}$ (213 cm e precisão de $0,1 \mathrm{~cm}$ ). 
O estado nutricional pré-gestacional e o ganho de peso semanal durante a gestação foram avaliados segundo os pontos de corte propostos pelo Institute of Medicine ${ }^{16}$. A classificação do estado nutricional atual das gestantes foi realizada de acordo com os valores estabelecidos por Atalah et al. ${ }^{17}$.

As análises estatísticas foram feitas no programa Stata, versão 12.0. Para a caracterização da população de estudo, as variáveis categóricas foram descritas por meio de frequências absolutas e relativas, e as quantitativas, por meio de medidas de tendência central e de dispersão.

A concentração de hemoglobina ( $\mathrm{g} / \mathrm{dL})$, selecionada como variável dependente, foi analisada como variável contínua. A associação entre as variáveis independentes estudadas e a concentração de hemoglobina foi verificada por meio de regressão linear múltipla.

Inicialmente, foram realizadas análises bivariadas para comparar as diferenças nas médias de hemoglobina entre as categorias das variáveis independentes, utilizando-se o teste $\mathrm{t}$ de Student, para variáveis dicotômicas, e a análise de variância (ANOVA), para variáveis com três ou mais categorias. As variáveis que apresentaram valor-P inferior a 0,20 na análise bivariada foram incluídas na análise de regressão linear multivariada, sendo a probabilidade inferior a $5 \%(\mathrm{P}<0,05)$ considerada como nível de significância estatística para a seleção no modelo de regressão múltipla.

Para a seleção do modelo final, utilizou-se a estratégia passo a passo, com a inclusão de todas as variáveis selecionadas durante a análise bivariada em ordem decrescente de significância estatística. As variáveis que apresentaram valor- $\mathrm{P}>0,05$ foram retiradas uma a uma do modelo e consideradas definitivamente excluídas, se o decréscimo na explicação do desfecho não era estatisticamente significativo. Para analisar esse parâmetro, o modelo foi avaliado a cada retirada com o auxílio do teste estatístico de Wald e coeficiente de determinação $\left(\mathrm{R}^{2}\right)$. Além disso, os modelos foram comparados por intermédio do critério de Akaike (AIC) para avaliação da qualidade de ajuste do modelo final.

O projeto de pesquisa foi submetido ao Comitê de Ética em Pesquisa da Universidade Estadual do Sudoeste da Bahia (Parecer $n^{\circ}$ 048/2010) e aprovado por ele. As gestantes diagnosticadas com anemia foram encaminhadas aos serviços de saúde do município para acompanhamento pelos profissionais de saúde e tratamento com sais de ferro, conforme a recomendação do Ministério da Saúde ${ }^{14}$.

\section{RESULTADOS}

Foi avaliada uma amostra de 328 gestantes, das quais a maioria era casada/com companheiro (86,9\%), tinha escolaridade igual ou superior a 8 anos $(57,6 \%)$ e estava na faixa etária de 20 a 35 anos (66,8\%), conforme Tabela 1 , sendo as médias de escolaridade e de idade iguais a 7,9 anos de estudo (desvio-padrão: 3,0) e 24 anos de idade (desvio-padrão: 6,2), respectivamente.

Em relação à condição socioeconômica, 85,3\% tinham renda familiar igual ou superior a um salário mínimo vigente na época do estudo (Tabela 1), sendo a mediana de renda familiar mensal igual a $\mathrm{R} \$ 542,50$.

No tocante às características obstétricas, $86,3 \%$ das gestantes avaliadas estavam no segundo ou terceiro trimestre gestacional, $27,7 \%$ eram primigestas e $37 \%$ iniciaram o pré-natal a partir da $14^{\text {a }}$ semana de gestação. O tabagismo e o consumo de álcool durante a gestação foram referidos por 6,1 e 5,2\% das gestantes, respectivamente (Tabela 2).

Tabela 1. Média e desvio-padrão (DP) de hemoglobina segundo características socioeconômicas e demográficas em gestantes atendidas nas unidades de saúde em Vitória da Conquista, Bahia, Brasil, 2011

\begin{tabular}{|c|c|c|c|}
\hline \multirow{2}{*}{ Variável / Categoria } & \multirow{2}{*}{$\mathbf{N}(\%)$} & \multicolumn{2}{|c|}{ Hemoglobina (g/dL) } \\
\hline & & Média (DP) & Valor-P \\
\hline \multicolumn{4}{|l|}{ Idade } \\
\hline$\leq 19$ anos & $92(28,05)$ & $12,07(1,17)$ & \multirow{3}{*}{0,3652} \\
\hline 20 a 35 anos & $219(66,77)$ & $11,87(1,19)$ & \\
\hline$>35$ anos & $17(5,18)$ & $11,80(1,08)$ & \\
\hline \multicolumn{4}{|l|}{ Situação conjugal } \\
\hline Solteira & $43(13,11)$ & $12,19(0,99)$ & \multirow[t]{2}{*}{0,1022} \\
\hline Casada/com companheiro & $285(86,89)$ & $11,88(1,20)$ & \\
\hline \multicolumn{4}{|l|}{ Escolaridade } \\
\hline$\geq 8$ anos de estudo & $189(57,62)$ & $11,85(1,20)$ & \multirow[t]{2}{*}{0,2082} \\
\hline$<8$ anos de estudo & $139(42,38)$ & $12,01(1,16)$ & \\
\hline \multicolumn{4}{|l|}{ Renda familiar } \\
\hline$\geq 1$ salário mínimo & $278(85,28)$ & $11,94(1,20)$ & \multirow[t]{2}{*}{0,5966} \\
\hline < 1 salário mínimo & $48(14,72)$ & $11,84(1,08)$ & \\
\hline \multicolumn{4}{|c|}{ Número de pessoas na família } \\
\hline$\leq 5$ pessoas & $274(83,54)$ & $11,94(1,19)$ & \multirow[t]{2}{*}{0,4575} \\
\hline$>5$ pessoas & $54(16,46)$ & $11,81(1,11)$ & \\
\hline
\end{tabular}


Tabela 2. Média e desvio-padrão (DP) de hemoglobina segundo características obstétricas e comportamentais em gestantes atendidas nas unidades de saúde em Vitória da Conquista, Bahia, Brasil, 2011

Variável / Categoria

\section{Idade gestacional}

Até a $13^{\text {a }}$ semana de gestação

$14^{\mathrm{a}}$ a $27^{\mathrm{a}}$ semana de gestação

N (\%)

$146(44,51)$

$\geq 28^{\text {a }}$ semana de gestação

$137(41,77)$

Início do pré-natal

Até a $13^{\text {a }}$ semana de gestação

$206(63,19)$

$120(36,81)$

$\geq 14^{\mathrm{a}}$ semana de gestação

Número de gestações

2 ou mais gestações

Primeira gestação

$91(27,74)$

$264(80,49)$

$64(19,51)$

$\geq 2$ filhos

Tabagismo na gestação

Não

$308(93,90)$

$20(6,10)$

Sim

Consumo de álcool na gestação

Não

$311(94,82)$

Sim
Hemoglobina (g/dL)

Média (DP) Valor-P

0,0006

$11,79(1,14)$

$11,85(1,11)$

$12,03(1,24)$

0,0312

$11,74(1,05)$

$11,82(1,16)$

0,0196

$12,16(1,20)$

$11,98(1,17)$

0,0712

$11,68(1,22)$

$11,90(1,18)$

0,3771

$12,15(1,18)$

$11,93(1,19)$

0,3206
Quanto ao estado nutricional, 27,2\% das mulheres tinham excesso de peso antes da gestação. Verificou-se também que apenas $4,3 \%$ das gestantes estavam com ganho de peso semanal adequado e $35,7 \%$ apresentavam sobrepeso ou obesidade na data da coleta de dados (Tabela 3).

No que diz respeito ao consumo alimentar, foi observado que a maioria das gestantes não consumia alimentos fontes de ferro, como vegetais folhosos verde-escuros $(78,7 \%)$ e carnes/aves/vísceras diariamente (71\%). Além disso, 26,8\% das gestantes não haviam sido suplementadas profilaticamente com sais de ferro (Tabela 3).

A prevalência de anemia verificada no estudo foi de 18,9\%, sendo a média de hemoglobina nas gestantes avaliadas igual a 11,9 g/dL (desvio-padrão: 1,2).

$\mathrm{Na}$ análise bivariada, observou-se diferença estatisticamente significativa $(P<0,05)$ entre as médias de hemoglobina nas categorias das variáveis idade gestacional, início do pré-natal, número de gestações, ganho de peso gestacional semanal e suplementação de ferro. Foram observadas menores médias de hemoglobina nas gestantes no segundo trimestre e que iniciaram o pré-natal a partir da $14^{\text {a }}$ semana de gestação; por outro lado, a maior média de hemoglobina foi constatada entre as primigestas (Tabela 2). As gestantes com ganho de peso semanal insuficiente e que não estavam recebendo suplemento de ferro também tiveram menores médias na concentração de hemoglobina (Tabela 3).

Além da idade gestacional, do início do pré-natal, do número de gestações, do ganho de peso gestacional semanal e da suplementação de ferro, as variáveis situação conjugal, número de filhos e consumo diário de feijão também foram incluídas na análise de regressão linear múltipla $(\mathrm{P}<0,20)$.

$\mathrm{Na}$ análise de regressão múltipla, as variáveis que se mostraram significativamente associadas com o desfecho foram: início do pré-natal, número de gestações e suplementação de ferro. As gestantes que iniciaram o pré-natal a partir do segundo trimestre tinham uma concentração de hemoglobina, em média, 0,28 g/dL menor, quando comparadas às gestantes que iniciaram o acompanhamento pré-natal no primeiro trimestre. As primigestas, por sua vez, tinham uma concentração de hemoglobina, em média, 0,34 g/dL maior, quando comparadas às mulheres que já tiveram outras gestações. A ausência de suplementação de ferro mostrou uma associação negativa com a concentração de hemoglobina. As gestantes que não foram suplementadas com sais de ferro tinham uma concentração de hemoglobina, em média, $0,51 \mathrm{~g} / \mathrm{dL}$ menor, em relação às que receberam suplementação de ferro (Figura 1). 
Tabela 3. Média e desvio-padrão (DP) de hemoglobina segundo características antropométricas, consumo de alimentos fontes de ferro e suplementação em gestantes atendidas nas unidades de saúde em Vitória da Conquista, Bahia, Brasil, 2011

\section{Variável / Categoria}

Índice de massa corporal pré-gestacional

Baixo peso

Eutrofia

Sobrepeso

Obesidade

N (\%)

$29(8,95)$

$207(63,89)$

$66(20,37)$

$22(6,79)$

Ganho de peso gestacional semanal

Insuficiente

Adequado

$170(52,47)$

$14(4,32)$

Excessivo

$140(43,21)$

Estado nutricional atual

Baixo peso

$64(19,51)$

Eutrofia

Sobrepeso

$147(44,82)$

$80(24,39)$

$37(11,28)$

Consumo diário de vegetais folhosos verde-escuros

Sim

$70(21,34)$

Não

$258(78,66)$

Consumo diário de feijão

Sim

$286(87,20)$

$42(12,80)$

Consumo diário de carnes/aves/vísceras

Sim

$95(28,96)$

$233(71,04)$

Suplementação de ferro

Sim

Não

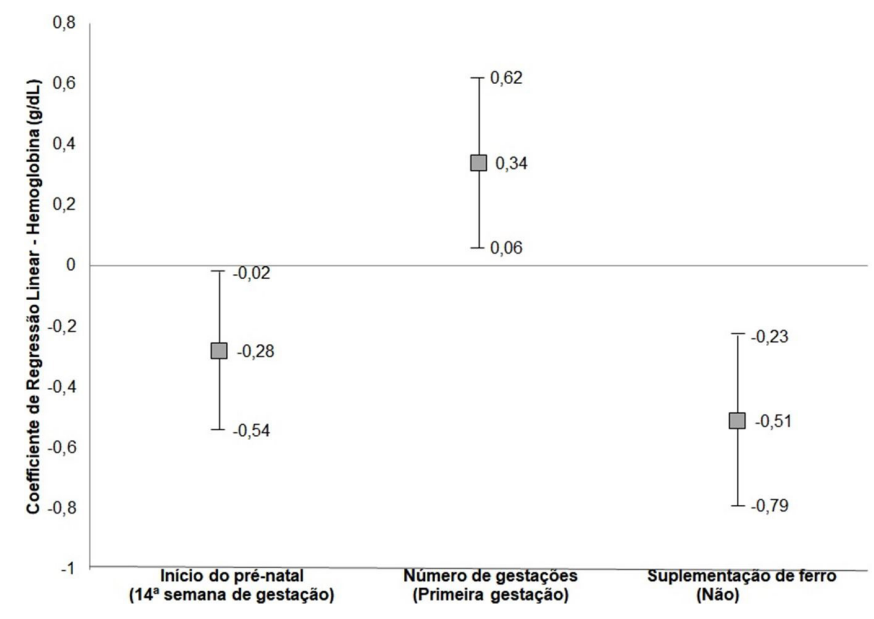

Figura 1. Análise de regressão linear múltipla para os determinantes da concentração de hemoglobina $(\mathrm{g} / \mathrm{dL})$ em gestantes atendidas nas unidades de saúde em Vitória da Conquista, Bahia, Brasil, 2011

\section{DISCUSSÃO}

No presente estudo, a prevalência de anemia nas gestantes avaliadas foi de $18,9 \%$, a qual, de acordo com a classificação proposta pela $\mathrm{OMS}^{18}$, é considerada como um leve problema de saúde pública. Porém, outros estudos realizados em
$240(73,17)$

$88(26,83)$
Hemoglobina (g/dL)

Média (DP) Valor-P

$11,74(1,33)$

$11,99(1,16)$

$11,69(1,19)$

$12,14(1,15)$

0,2032

$11,85(1,20)$

$12,83(1,24)$

0,0116

$11,91(1,13)$

$11,76(1,12)$

$11,99(1,17)$

0,6049

$11,89(1,32)$

$11,98(1,04)$

$11,89(1,08)$

0,8464

$11,93(1,21)$

$11,95(1,15)$

0,1850

$11,69(1,37)$

$11,84(1,06)$

0,4547

$11,95(1,23)$

$12,29(1,31)$

$11.78(1,10)$ municípios da região Nordeste têm observado prevalências superiores. No estudo realizado por Ferreira et al. ${ }^{19}$, na região semiárida do Estado de Alagoas, observou-se uma prevalência de $50 \%$ de anemia nas gestantes estudadas, enquanto os resultados da pesquisa realizada por Bresani et al.$^{20} \mathrm{em}$ Recife, Pernambuco, mostraram que $56,6 \%$ das gestantes avaliadas estavam anêmicas.

Embora a coleta de dados do estudo de Bresani e colaboradores ${ }^{20}$ tenha ocorrido antes do ano de 2004, em que se tornou obrigatória a fortificação das farinhas de trigo com ferro e ácido fólico ${ }^{21}$, alguns estudos têm demonstrado que a fortificação não implicou aumento significativo nos níveis de hemoglobina em gestantes ${ }^{22}$ e que, mesmo após essa intervenção, a prevalência de anemia em gestantes, na maioria dos municípios da região Nordeste, ainda permanece elevada ${ }^{23}$. Além disso, é importante destacar que, no estudo de Ferreira et al. ${ }^{19}$, verificou-se que apenas $21,2 \%$ das gestantes faziam uso de suplementos de ferro. Dessa forma, supõe-se que não a fortificação das farinhas, mas a maior frequência de suplementação de profilática com sais de ferro $(73,2 \%)$ nas gestantes do município de Vitória da Conquista pode ter contribuído, em parte, para uma menor prevalência de anemia no presente estudo. 
A média da concentração sérica de hemoglobina nas gestantes avaliadas foi de 11,9 $\mathrm{g} / \mathrm{dL}$, bem próximo ao valor mínimo considerado como normal durante a gestação. A dosagem de hemoglobina é fundamental no período gestacional, estando incluída entre os exames que devem ser solicitados na primeira consulta de pré-natal ${ }^{14}$. O seu monitoramento é importante não apenas para a prevenção e o controle da anemia, mas também para evitar o risco de morte materna e infantil. Uma metanálise mostrou que, para cada $1 \mathrm{~g} / \mathrm{dL}$ de aumento na média da concentração de hemoglobina durante a gestação, há uma diminuição de 20 e 16\% do risco de mortalidade materna e perinatal, respectivamente ${ }^{24}$.

Em relação aos determinantes da concentração sérica de hemoglobina, observaram-se menores concentrações de hemoglobina sérica entre as gestantes que iniciaram o acompanhamento pré-natal após a $13^{\text {a }}$ semana de gestação. Um achado semelhante foi reportado no estudo de Ferreira et al. ${ }^{19}$, no qual se verificou uma maior prevalência de anemia entre as gestantes que iniciaram o pré-natal após o primeiro trimestre de gestação. O Ministério da Saúde ${ }^{14}$ recomenda que a primeira consulta pré-natal deva ocorrer ainda no primeiro trimestre gestacional, bem como devem ser realizadas no mínimo seis consultas até o final da gestação. $\mathrm{O}$ início do acompanhamento pré-natal no período recomendado aumenta as chances de realização de um maior número de consultas e, consequentemente, menores riscos de agravos à saúde da mãe e do concepto.

Outro fator determinante da concentração de hemoglobina verificado neste estudo foi o número de gestações, sendo observadas maiores concentrações séricas de hemoglobina entre as gestantes que estavam em sua primeira gestação. Em contrapartida, Guerra et al. ${ }^{25}$ não encontraram diferença significativa entre as médias das concentrações de hemoglobina das gestantes primigestas e multigestas. Embora esse achado não tenha sido reportado em outros estudos na literatura, uma hipótese que poderia suportar a associação observada no presente estudo é que as mulheres primigestas, por estarem vivenciando uma experiência nova, tendem a estar mais preocupadas com a saúde durante a gestação. Assim, essas mulheres tornam-se mais cuidadosas em relação à alimentação, à realização das consultas, aos exames e ao uso de suplementos durante o pré-natal, o que favorece a manutenção da hemoglobina sérica em concentrações adequadas.

A suplementação com sais de ferro também foi outro fator determinante da concentração de hemoglobina nas gestantes. Observaram-se menores médias de hemoglobina entre as gestantes que não fizeram uso de suplemento de ferro. Sugere-se que o alto requisito desse mineral durante a gravidez associada à não suplementação pode ter resultado em concentrações mais baixas de hemoglobina.

A suplementação de ferro é a estratégia mais utilizada para tratar a anemia ferropriva e também deve ser considerada como uma medida preventiva de saúde pública para controlar a deficiência de ferro em populações com alto risco de desenvolvimento de anemia ${ }^{18}$. O Ministério da Saúde ${ }^{26}$, por meio do Programa Nacional de Suplementação de Ferro, que objetiva a prevenção e o controle da anemia de crianças de 6 a 24 meses, gestantes e mulheres até o $3^{\circ}$ mês pós-parto e/ou pós-aborto, recomenda a administração profilática diária de $40 \mathrm{mg}$ de ferro elementar para garantir a manutenção das reservas de ferro e suprir os requerimentos gestacionais. Apesar da existência desse programa e de tais recomendações, observa-se que ainda há gestantes que não são suplementadas com sais de ferro durante a gestação. Essa lacuna na cobertura do programa pode estar relacionada a fatores, como a indisponibilidade do suplemento nos serviços, a sua não distribuição por parte dos funcionários ou a distribuição sem as devidas orientações, o não comparecimento das gestantes às consultas ou a sua não adesão à prescrição ${ }^{19}$.

Algumas limitações deste estudo são apontadas. O estudo é de delineamento transversal, o que não permite o estabelecimento de uma relação temporal entre algumas variáveis de exposição com o desfecho. O diagnóstico da anemia foi realizado pelo método Cianometahemoglobina, utilizando o Hemocue ${ }^{\circledR}$, o qual avalia apenas o nível de hemoglobina e pode resultar em diagnóstico falso-negativo. Porém, o uso desse método é validado para pesquisas de campo e tem sido amplamente utilizado em pesquisas epidemiológicas. Além disso, apresenta especificidade e sensibilidade suficientes para detectar níveis alterados de hemoglobina ${ }^{27}$.

Como pontos fortes, destaca-se que o estudo traz uma grande contribuição para a literatura, considerando a escassez de pesquisas nessa população sobre o tema nos últimos anos. Além disso, o estudo avaliou os determinantes da concentração de hemoglobina, em vez da anemia propriamente dita, de forma a identificar fatores que influenciam as concentrações séricas de hemoglobina na gestação, possibilitando intervir na situação antes mesmo de o quadro de anemia ser instalado.

Os resultados do presente estudo mostram que a anemia nas gestantes assistidas em unidades de saúde da zona urbana do município de Vitória da Conquista é um leve problema de saúde pública. As concentrações de hemoglobina nessa população são determinadas por fatores obstétricos e relacionados à assistência pré-natal. Nesse contexto, destaca-se a importância da atenção básica no cuidado à saúde da gestante, o que inclui desde o acesso à educação em saúde até a disponibilização de exames complementares e suplementos/medicamentos no cuidado a agravos de saúde, como a anemia. Ressalta-se que os estudos sobre o tema são de grande relevância, uma vez que seus resultados podem direcionar à implementação de medidas de saúde pública visando à manutenção e ao controle da concentração de hemoglobina em níveis adequados e à prevenção da anemia na gestação. 


\section{REFERÊNCIAS}

1. Carvalho MC, Baracat ECE, Sgarbieri VC. Anemia ferropriva e anemia de doença crônica: distúrbios do metabolismo de ferro. Segur Aliment Nutr. 2006;13(2):54-63. http://dx.doi.org/10.20396/san.v13i2.1832.

2. Brasil. Ministério da Saúde. Carências de micronutrientes. Brasília: Ministério da Saúde; 2007. (Cadernos de Atenção Básica; 20).

3. Fundo das Nações Unidas para a Infância. The State of the World's Children 1998: a UNICEF report malnutrition: causes, consequences, and solutions. Nutr Rev. 1998;56(4 Pt 1):115-23. PMid:9584496.

4. Souza AI, Batista M Fo, Ferreira LOC. Alterações hematológicas e gravidez. Rev Bras Hematol Hemoter. 2002;24(1):29-36. http://dx.doi.org/10.1590/ S1516-84842002000100006.

5. Viteri FE. The consequences of iron deficiency and anaemia in pregnancy on maternal health the foetus and the infant. Hemoglobin. 1997;90(310):250.

6. Allen LH. Anemia and iron deficiency: effects on pregnancy outcome Am J Clin Nutr. 2000;71(5 Suppl):1280s-4s. http://dx.doi.org/10.1093/ ajen/71.5.1280s. PMid:10799402.

7. World Health Organization. Worldwide prevalence of anaemia 1993-2005: WHO global database on anaemia. Geneva: WHO; 2008.

8. Souza AI, Batista M Fo. Diagnóstico e tratamento das anemias carenciais na gestação: consensos e controvérsias. Rev Bras Saude Mater Infant. 2003;3(4):473-9. http://dx.doi.org/10.1590/S1519-38292003000400012.

9. Côrtes MH, Vasconcelos IAL, Coitinho DC. Prevalência de anemia ferropriva em gestantes brasileiras: uma revisão dos últimos 40 anos. Rev Nutr. 2009;22(3):409-18. http://dx.doi.org/10.1590/S1415-52732009000300011.

10. Instituto Brasileiro de Geografia e Estatística. Vitória da Conquista - BA [Internet]. Rio de Janeiro: IBGE; 2010 [citado em 2012 dez 28]. Disponível em: http://tabnet.datasus.gov.br/tabdata/cadernos/ba.htm

11. Instituto Brasileiro de Geografia e Estatística.Cidades@: Bahia - Vitória da Conquista - Síntese das informações. Rio de Janeiro: IBGE; 2015 [Internet]. [citado em 2015 fev 10]. Disponível em: http://cidades.ibge.gov.br/xtras/ temas.php?lang $=\& \operatorname{codmun}=293330$ \&idtema $=16 \&$ search $=||$ s\%EDntesedas-informa\%E7\%F5es

12. Brasil. Ministério da Saúde. Cadernos de Informações de Saúde - Bahia [Internet]. Rio de Janeiro: IBGE; 2018 [citado em 2018 ago. 17]. Disponível em: http://www.ibge.gov.br/cidadesat/painel/painel.php?codmun=293330

13. Sato APS. Anemia em gestantes atendidas em serviços públicos de prénatal das cinco regiões brasileiras antes e após a política da fortificação das farinhas com ferro [dissertação]. São Paulo: Universidade de São Paulo; 2010. http://dx.doi.org/10.11606/D.7.2010.tde-13012011-154115.

14. Brasil. Ministério da Saúde. Pré-natal e puerpério: atenção qualificada e humanizada. Brasília, DF: Ministério da Saúde; 2006.

15. Brasil. Ministério da Saúde. Orientações para a coleta e análise de dados antropométricos em serviços de saúde: Norma Técnica do Sistema de
Vigilância Alimentar e Nutricional -SISVAN. Brasília, DF: Ministério da Saúde; 2011.

16. Institute of Medicine. Weight gain during pregnancy: reexamining the guidelines. Kathmandu: IOM; 2009.

17. Atalah E, Castillo C, Castro R, Aldea A. Propuesta de un nuevo estándar de evaluación nutricional en embarazadas. Rev Med Chil. 1997;125(12):142936. PMid:9609018.

18. World Health Organization. Iron deficiency Anaemia. Assessment, Prevention and Control. A guide programme managers. Geneva: WHO; 2001.

19. Ferreira HS, Moura FA, Cabral CR Jr. Prevalência e fatores associados à anemia em gestantes da região semi-árida do Estado de Alagoas. Rev Bras Ginecol Obstet. 2008;30(9):445-51. http://dx.doi.org/10.1590/S010072032008000900004. PMid:19142530.

20. Bresani CC, Souza BAI, Batista M Fo, Figueiroa JN. Anemia e ferropenia em gestantes: dissensos de resultados de um estudo transversal. Rev Bras Saude Mater Infant. 2007;7(Supl. 1):s15-21. http://dx.doi.org/10.1590/ S1519-38292007000600002.

21. Brasil. Agência Nacional de Vigilância Sanitária. Resolução RDC n ${ }^{\circ} 344$ de 13 de dezembro de 2002. Regulamento técnico para a fortificação das farinhas de trigo e das farinhas de milho com ferro e ácido fólico. Diário Oficial da União, Brasília, 18 de dezembro de 2002.

22. Sato APS, Fujimori E, Szarfarc SC, Sato JR, Bonadio IC. Prevalência de anemia em gestantes e a fortificação de farinhas com ferro. Texto Contexto Enferm. 2008;17(3):474-81. http://dx.doi.org/10.1590/S0104-07072008000300008.

23. Fujimori E, Sato AP, Szarfarc SC, Veiga GV, Oliveira VA, Colli C. et al. Anemia em gestantes brasileiras antes e após a fortificação das farinhas com ferro. Rev Saude Publica. 2011;45(6):1027-35. http://dx.doi.org/10.1590/ S0034-89102011005000078. PMid:22127652.

24. Stoltzfus RJ. Iron deficiency: global prevalence and consequences. Food Nutr Bull. 2003;24(4 Suppl):S99-103. http://dx.doi.org/10.1177/15648265030244S206. PMid:17016951.

25. Guerra EM, Barretto OCDO, Vaz AJ, Silveira MB. The prevalence of anemia in first consultation pregnant women of health centers in a metropolitan area, Brazil. Rev Saude Publica. 1990;24(5):380-6. http://dx.doi.org/10.1590/ S0034-89101990000500005. PMid:2101529.

26. Brasil. Ministério da Saúde. Portaria nº. 730, de 13 de maio de 2005. Institui o Programa Nacional de Suplementação de Ferro, destinado a prevenir a anemia ferropriva e dá outras providências. Diário Oficial da União, Brasília, 16 de maio de 2005

27. Jordão RE, Bernardi JLD, Barros AA Fo. Prevalência de anemia ferropriva no Brasil: uma revisão sistemática. Rev Paul Pediatr. 2009;27(1):90-8. http:// dx.doi.org/10.1590/S0103-05822009000100014.

Recebido em: Fev. 28, 2018 Aprovado em: Set. 04, 2018 